\title{
COMPACT LINEAR OPERATORS FROM AN ALGEBRAIC STANDPOINT
}

\author{
by F. F. BONSALL \\ (Received 11 February, 1966)
}

\begin{abstract}
1. Introduction. Let $B(X)$ denote the Banach algebra of all bounded linear operators on a Banach space $X$. Let $t$ be an element of $B(X)$, and let $e$ denote the identity operator on $X$. Since the earliest days of the theory of Banach algebras, it has been understood that the natural setting within which to study spectral properties of $t$ is the Banach algebra $B(X)$, or perhaps a closed subalgebra of $B(X)$ containing $t$ and $e$. The effective application of this method to a given class of operators depends upon first translating the data into terms involving only the Banach algebra structure of $B(X)$ without reference to the underlying space $X$. In particular, the appropriate topology is the norm topology in $B(X)$ given by the usual operator norm. Theorem 1 carries out this translation for the class of compact operators $t$. It is proved that if $t$ is compact, then multiplication by $t$ is a compact linear operator on the closed subalgebra of $B(X)$ consisting of operators that commute with $t$.

In $\S 3$ we exploit Theorem 1 by showing how the Riesz-Schauder spectral theory for a compact linear operator $t$ may be obtained by applying the most elementary Banach algebra techniques to the least closed subalgebra of $B(X)$ containing $t$ and $e$.

As a second application of Theorem 1, we prove a theorem which contains the KreinRutman theorem [5, Theorem 6.1] on positive compact linear operators. Let $t$ be compact and have non-zero spectral radius $\rho$, and let $A^{+}$denote the least closed semi-algebra in $B(X)$ containing $t$ and $e$. Using entirely elementary arguments together with Theorem 1 , we prove that if $A^{+} \cap\left(-A^{+}\right)=(0)$, then there exists a nonzero element $u$ of $A^{+}$such that $t u=\rho u$. This result gives the Krein-Rutman theorem at once.

Theorem 1 may be regarded as an analogue of Schauder's theorem [8] on the compactness of the adjoint of a compact linear operator, and we give in $\S 5$ a theorem which includes both Theorem 1 and Schauder's theorem as special cases. As another special case of this theorem, we see that, if $t$ is compact, the mapping $a \rightarrow$ tat is a compact linear operator on the whole of $B(X)$. This result is fundamental for the recent work of J. C. Alexander [1].
\end{abstract}

\section{The compactness of multiplication operators.}

THEOREM 1. Let $t$ be a compact linear operator on a Banach space $X$, and let $Y$ be the centralizer of $t$. Then the mapping $a \rightarrow t a(a \in Y)$ is a compact linear operator on $Y$.

Proof. By definition, the centralizer $Y$ of $t$ is the set of all bounded linear operators that commute with $t$. It is clear that $Y$ is a closed subalgebra of $B(X)$ and that the mapping $a \rightarrow t a$ is a bounded linear operator on the Banach space $Y$.

Let $X_{1}$ denote the closed unit ball in $X$, and let $E=\overline{t X_{1}}$. Then $E$ is a compact subset of $X$ in the norm topology. Given $a \in Y$ with $\|a\| \leqq 1$, we have at $X_{1}=\operatorname{ta} X_{1} \subset t X_{1}$; and there- 
fore, by continuity, $a E \subset E$. Let $a_{n} \in Y,\left\|a_{n}\right\| \leqq 1(n=1,2, \ldots)$. Then, for each $x \in E$, the set $\left\{a_{n} x: n=1,2, \ldots\right\}$ is contained in the compact subset $E$ of the Banach space $X$. Also

$$
\left\|a_{n} x-a_{n} x^{\prime}\right\| \leqq\left\|x-x^{\prime}\right\| \quad\left(x, x^{\prime} \in E, n=1,2, \ldots\right),
$$

which shows that the mappings $x \rightarrow a_{n} x(x \in E, n=1,2, \ldots)$ form an equicontinuous sequence of mappings of the compact space $E$ into the Banach space $X$. By Ascoli's theorem for Banach space valued functions, it follows that there exists a subsequence $\left\{a_{n_{k}}\right\}$ such that $\left\{a_{n_{k}} x\right\}$ converges uniformly for $x$ in $E$. Consequently, $\left\{a_{n_{k}} t x\right\}$ converges uniformly for $x$ in $X_{1}$, and so $\left\{a_{n_{k}} t\right\}$ converges with respect to the operator norm. Since $a_{n_{k}} t \in Y$ and $Y$ is closed, this shows that $\left\{a_{n_{k}} t\right\}$ converges in $Y$. Finally, $t a_{n}=a_{n} t$.

Counter-example. Let $X$ have infinite dimension, let $A$ be a strictly irreducible subalgebra of $B(X)$, and let $t$ be a nonzero element of $A$. Then the linear mapping $a \rightarrow a t(a \in A)$ is not compact on $A$. For, since $X$ is an infinite dimensional normed space, there exist elements $x_{n}$ of $X$ such that $\left\|x_{n}\right\|=1(n=1,2, \ldots)$ and $\left\|x_{k}-x_{j}\right\| \geqq \frac{1}{2}(k \neq j)$. Since $t \neq 0$, there exists $x_{0} \in X$ with $t x_{0} \neq 0$. Then $t x_{0}$ is a strictly cyclic vector, and so (see [4], Proposition 17, Corollary 1) there exist a constant $M$ and elements $a_{n}$ of $A$ such that

$$
\left\|a_{n}\right\| \leqq M, \quad a_{n} t x_{0}=x_{n} \quad(n=1,2, \ldots) .
$$

If the mapping $a \rightarrow a t(a \in A)$ is compact, there exists a subsequence $\left\{a_{n_{k}}\right\}$ such that $\left\{a_{n_{k}} t\right\}$ converges. But then $\left\{x_{n_{k}}\right\}$ converges, which is absurd.

Similarly, if the algebra $A^{*}$ (the set of adjoints of elements of $A$ ) is strictly irreducible on the dual space $X^{*}$ of $X$, then the mapping $a \rightarrow t a(a \in A)$ is not compact. For, by what we have just proved, the mapping $a^{*} \rightarrow a^{*} t^{*}\left(a^{*} \in A^{*}\right)$ is not compact, and the mapping $a \rightarrow a^{*}$ is an isometric anti-isomorphism of $A$ on to $A^{*}$.

If $A$ is dually strictly irreducible [4] on the pair of spaces $X, X^{*}$, then neither of the mappings $a \rightarrow a t, a \rightarrow t a$ is compact on $A$. This is the case in particular for $A=B(X)$ or for any subalgebra $A$ of $B(X)$ that contains all operators of finite rank.

3. Riesz-Schauder theory. We need to make use of two elementary propositions from the usual theory of compact operators [6].

Proposition 1. Let $t$ be a compact linear operator on a Banach space $X$, and let $\left\{\lambda_{n}\right\}$ be $a$ sequence of distinct eigenvalues of $t$. Then

$$
\lim _{n \rightarrow \infty} \lambda_{n}=0
$$

Proposition 2. Let $t$ be a compact linear operator on a Banach space $X, \lambda$ a nonzero eigenvalue of $t$, and let

$$
N_{k}=\left\{x:(t-\lambda e)^{k} x=0\right\} \quad(k=1,2, \ldots) .
$$

Then each $N_{k}$ has finite dimension, and there exists a positive integer $k$ such that $N_{k+1}=N_{k}$. 
The well known proofs of both propositions depend on the fact that if $X_{0}$ is a proper closed linear subspace of $X$, then there exists $x \in X$ such that $\|x\|=1$ and $\|x-y\| \geqq \frac{1}{2}$ $\left(y \in X_{0}\right)$.

For Theorems 2 and 3, we shift our attention to an abstract Banach algebra.

Notation. $A$ will denote a commutative Banach algebra with unit element 1 . We suppose that $A$ contains an element $t$ such that the mapping $a \rightarrow t a$ is a compact linear operator on the Banach space $A$. We denote the spectrum of an element $a$ of $A$ by $\operatorname{Sp}_{A}(a)$, i.e. $\operatorname{Sp}_{A}(a)$ is the set of complex numbers $\lambda$ such that $\lambda-a$ has no inverse in $A$.

Lemma 1. Let $\lambda$ be a nonzero frontier point of $\mathrm{Sp}_{A}(t)$. Then there exists $u \in A$ such that $u \neq 0$ and $t u=\lambda u$.

Proof. $t-\lambda$ is a frontier point of the set of invertible elements of $A$, and is therefore a topological divisor of zero [7, Theorem 1.5.4]. Thus there exist elements $a_{n}$ of $A$ such that $\left\|a_{n}\right\|=1(n=1,2, \ldots)$, and $\lim _{n \rightarrow \infty}(t-\lambda) a_{n}=0$. Since the mapping $a \rightarrow t a$ is compact, there exists a subsequence $\left\{a_{n_{k}}\right\}$ such that $\lim _{k \rightarrow \infty} t a_{n_{k}}=u$, say. Since $\lim _{k \rightarrow \infty}\left(t a_{n_{k}}-\lambda a_{n_{k}}\right)=0$, we have in turn $\lim _{k \rightarrow \infty} \lambda a_{n_{k}}=u, \lim _{k \rightarrow \infty} \lambda t a_{n_{k}}=t u, t u=\lambda u$. Also, $\|u\|=\lim _{k \rightarrow \infty}|\lambda|\left\|a_{n_{k}}\right\|=|\lambda|$, and so $u \neq 0$.

THEOREM 2. 0 is the only possible point of accumulation of $\operatorname{Sp}_{A}(t)$; and if $\lambda \in \operatorname{Sp}_{A}(t)$ and $\lambda \neq 0$, then there exists $u \in A$ such that $u \neq 0$ and $t u=\lambda u$.

Proof. Lemma 1 and Proposition 1 show that 0 is the only possible point of accumulation of the frontier of $\operatorname{Sp}_{A}(t)$. Since $\operatorname{Sp}_{A}(t)$ is a compact subset of the complex plane, it follows that 0 is the only possible point of accumulation of $\mathrm{Sp}_{A}(t)$, and so all points of $\mathrm{Sp}_{A}(t)$ are frontier points. A second application of Lemma 1 completes the proof.

Notation. For Theorem 3, we suppose that $\lambda \in \operatorname{Sp}_{A}(t)$ and that $\lambda \neq 0$, and we define $N_{k}, R_{k}$ by

$$
N_{k}=\left\{a \in A:(t-\lambda)^{k} a=0\right\}, \quad R_{k}=(t-\lambda)^{k} A .
$$

By Proposition 2, there exists a least positive integer $v$ (the index of $\lambda$ ) such that $N_{v+1}=N_{v}$. It is easly verified that in fact $N_{k}=N_{v}(k \geqq v)$.

THEOREM 3. (i) $A=N_{v} \oplus R_{v}$.

(ii) $1=p+q$, where $p$ and $q$ are idempotents that are unit elements for the subalgebras $N_{v}$ and $R_{v}$ respectively.

(iii) $(t-\lambda) q$ is invertible relative to the subalgebra $R_{v}$, i.e. there exists $u \in R_{v}$ such that $(t-\lambda) q u=q$.

(iv) $(t-\lambda) R_{v}=R_{v}$.

(v) $v$ is the least positive integer for which $(t-\lambda)^{v+1} A=(t-\lambda)^{v} A$. 
Proof. (i) By a standard algebraic argument, we have $N_{v} \cap R_{v}=(0)$. For if $a \in N_{v} \cap R_{v}$, then $a=(t-\lambda)^{v} b$ for some $b \in A$. Then $(t-\lambda)^{2 v} b=0$, and so $b \in N_{2 v}=N_{v}, a=0$.

$N_{v}$ is a closed ideal of $A$. Let $B$ denote the difference algebra $A-N_{v}$, the elements of which are the cosets $a^{\prime}=a+N_{v}$. With the canonical norm $\left\|a^{\prime}\right\|=\inf \left\{\|x\|: x \in a^{\prime}\right\}, B$ is a commutative Banach algebra with unit element $1^{\prime}$. We prove next that the mapping $a^{\prime} \rightarrow t^{\prime} a^{\prime}$ is a compact linear operator on $B$. In fact, given $b_{n} \in B$ with $\left\|b_{n}\right\| \leqq 1(n=1,2, \ldots)$, there exist elements $a_{n} \in A$ with $\left\|a_{n}\right\| \leqq 2$ and $a_{n}{ }^{\prime}=b_{n}$. Then there exists a subsequence $\left\{a_{n_{k}}\right\}$ such that $\lim _{k \rightarrow \infty} t a_{n_{k}}=a$, say, and therefore $\lim _{k \rightarrow \infty} t^{\prime} b_{n_{k}}=\lim _{k \rightarrow \infty}\left(t a_{n_{k}}\right)^{\prime}=a^{\prime}$. This shows that Theorem 2 is applicable to $B$ and $t^{\prime}$ in place of $A$ and $t$, and it follows that $\lambda \notin \mathrm{Sp}_{B}\left(t^{\prime}\right)$. For otherwise there exists $u \in A$ such that $u^{\prime} \neq 0$ and $t^{\prime} u^{\prime}=\lambda u^{\prime}$. But then $(t-\lambda) u \in N_{v}, u \in N_{v+1}=N_{v}, u^{\prime}=0$, a contradiction. Therefore $(t-\lambda)^{\prime}$ has an inverse $a^{\prime}$, say, in $B$, i.e. $(t-\lambda)^{\prime} a^{\prime}=1^{\prime}$. But then $\left((t-\lambda)^{v} a^{v}\right)^{\prime}=1^{\prime}$, and so there exists $v \in N_{v}$ such that $1=v+(t-\lambda)^{v} a^{v}$. This shows that $1 \in N_{v} \oplus R_{v}$, and, since $N_{v} \oplus R_{v}$ is an ideal, we have $A=N_{v} \oplus R_{v}$.

(ii) This is clear. We merely decompose 1 into its components $p$ and $q$ in $N_{v}$ and $R_{v}$ respectively.

(iii) We have seen that $(t-\lambda)^{\prime}$ is invertible in $B=A-N_{v}$. However, the canonical mapping $a \rightarrow a^{\prime}$ is an isomorphism of $R_{v}$ on to $B$, and $((t-\lambda) q)^{\prime}=(t-\lambda)^{\prime}$. Therefore $(t-\lambda) q$ is invertible relative to the subalgebra $R_{v}$, i.e. there exists $u \in R_{v}$ such that $(t-\lambda) q u=q$.

(iv) $(t-\lambda) R_{v}=(t-\lambda) q R_{v}=R_{v}$.

(v) We have $(t-\lambda)^{v+1} A=(t-\lambda) R_{v}=R_{v}=(t-\lambda)^{v} A$. Also, if $k$ is a positive integer for which $(t-\lambda)^{k+1} A=(t-\lambda)^{k} A$, then $(t-\lambda)^{k}=(t-\lambda)^{k+1} a$ for some $a \in A$. Therefore, if $(t-\lambda)^{k+1} x=0$, we have $(t-\lambda)^{k} x=(t-\lambda)^{k+1} a x=0$, and so $k \geqq v$.

We now apply Theorems 2 and 3 to obtain spatial properties of a bounded linear operator $t$ on a Banach space $X$. We denote the spectrum of $t$ by $\operatorname{Sp}(t)$, i.e. $\operatorname{Sp}(t)=\operatorname{Sp}_{B(X)}(t)$.

THeOREM 4. Let $t \in B(X)$, let $A$ be the least closed subalgebra of $B(X)$ containing $t$ and $e$, and suppose that the mapping $a \rightarrow \operatorname{ta}(a \in A)$ is a compact linear operator on $A$. Then the following propositions hold.

(i) $\mathrm{Sp}(t)=\mathrm{Sp}_{A}(t)$, and each nonzero point of $\mathrm{Sp}(t)$ is an eigenvalue of $t$.

(ii) Let $\lambda$ be a nonzero eigenvalue of $t$, let $p, q$ be the projections given in Theorem 3 , and let $Y=p X, Z=q X$. Then $Y, Z$ are closed invariant subspaces for $t$,

$$
Y=\left\{x:(t-\lambda e)^{v} x=0\right\}, Z=(t-\lambda e)^{v} X,
$$

$X=Y \oplus Z$, the restriction of $t-\lambda$ e to $Z$ is invertible in $B(Z)$, and the index of $\lambda$ for the linear operator $t$ is equal to its index $v$ for $t$ regarded as an element of $A$.

(iii) Let $t^{*}$ denote the adjoint of $t$, and $A^{*}$ the least closed subalgebra of $B\left(X^{*}\right)$ containing $t^{*}$ and $e^{*}$. Then the mapping $a^{*} \rightarrow t^{*} a^{*}\left(a^{*} \in A^{*}\right)$ is a compact linear operator on $A^{*}$, and propositions (i) and (ii) hold for $t^{*}, A^{*}, X^{*}$ in place of $t, A, X$. The index of a nonzero eigenvalue for the linear operator $t^{*}$ is equal to its index for $t$, and for $k=0,1, \ldots, v-1$, the ranges of $(t-\lambda e)^{k} p$ and $\left(t^{*}-\lambda e^{*}\right)^{k} p^{*}$ have equal dimension. 
Remarks. (i) Theorem 1 shows that Theorem 4 is applicable in particular to a compact linear operator $t$ on $X$.

(ii) The spectral projection $p$ is an element of $A$. In fact, it belongs to the least closed subalgebra $A_{0}$ of $B(X)$ that contains $t$. For we have $(t-\lambda e)^{v} p=0$, and $\lambda \neq 0$, from which $p \in t A \subset A_{0}$.

(iii) Theorem 4 is applicable to operators $t$ that are not compact on $X$; for example the identity operator $e$.

Proof. (i) Since $A \subset B(X)$, it is obvious that $\mathrm{Sp}(t) \subset \mathrm{Sp}_{A}(t)$, and since all points of $\mathrm{Sp}_{A}(t)$ are frontier points, $\mathrm{Sp}_{A}(t) \subset \mathrm{Sp}(t)$, [7, Theorem 1.6.12]. It is now clear from Theorem 2 that all nonzero points of $\mathrm{Sp}(t)$ are eigenvalues.

(ii) It is clear that $Y$ and $Z$ are closed invariant subspaces for $t$ and that $X=Y \oplus Z$. By Theorem 3(iii), $(t-\lambda e) q$ is invertible relative to $R_{v}$, and so $\left.(t-\lambda e)\right|_{z}$ is invertible in $B(Z)$. We note next that

$$
Y=\left\{x \in X:(t-\lambda e)^{v} x=0\right\}, \quad Z=(t-\lambda e)^{v} X .
$$

For, since $q A=R_{v}=(t-\lambda e)^{v} A$, there exist $a, b \in A$ with

$$
q=a(t-\lambda e)^{\nu}, \quad(t-\lambda e)^{\nu}=q b .
$$

It follows at once from (2) that $(t-\lambda e)^{v} X=q X$ and that $(t-\lambda e)^{v} x=0$ if and only if $q x=0$, which proves (1).

We prove that $v$ is the least positive integer $k$ for which

$$
\left\{x \in X:(t-\lambda e)^{k+1} x=0\right\}=\left\{x \in X:(t-\lambda e)^{k} x=0\right\} .
$$

For if $k$ satisfies (3) and $(t-\lambda e)^{k+1} a=0$ for some $a \in A$, then we have in turn $(t-\lambda e)^{k+1} a x=0$ $(x \in X),(t-\lambda e)^{k} a x=0(x \in X),(t-\lambda e)^{k} a=0$; which shows that $k \geqq v$. On the other hand, if $(t-\lambda e)^{v+1} x=0$ for some $x \in X$, then $(t-\lambda e)^{2 v} x=0$; and, by (1), $(t-\lambda e)^{v} x \in Y \cap Z,(t-\lambda e)^{v} x$ $=0$. Thus $v$ is an integer satisfying (3).

(iii) The mapping $a \rightarrow a^{*}$ of $a$ on to its adjoint $a^{*}$ is an isometric isomorphism between $A$ and $A^{*}$. Thus the properties of $\left(t^{*}, A^{*}, X^{*}\right)$ can be deduced from those of $(t, A, X)$. The final statement is an elementary consequence of the duality between $X$ and $X^{*}$. Let $a=(t-\lambda e)^{k} p$, and let $x_{1}, \ldots, x_{n}$ be linearly independent elements of $a X$. Then there exist $f_{1}, \ldots, f_{n} \in X^{*}$ and $y_{1}, \ldots, y_{n} \in X$ such that $f_{i}\left(x_{j}\right)=\delta_{i j}$ and $x_{j}=a y_{j}(i, j=1, \ldots, n)$. Then

$$
\left(a^{*} f_{i}\right)\left(y_{j}\right)=f_{i}\left(a y_{j}\right)=f_{i}\left(x_{j}\right)=\delta_{i j},
$$

showing that $a^{*} f_{1}, \ldots, a^{*} f_{n}$ are linearly independent elements of $a^{*} X^{*}$. Thus the dimension of $a X$ does not exceed the dimension of $a^{*} X^{*}$, and similarly for the opposite inequality.

4. The Krein-Rutman theorem. We show how Theorem 1 may be applied to give a new proof of the following theorem of M. G. Krein and M. A. Rutman [5, Theorem 6.1]. 
THEOREM. Let $X^{+}$be a closed cone in a Banach space $X$ such that $X$ is the closed linear hull of $X^{+}$. Let $t$ be a compact linear operator on $X$ such that $t X^{+} \subset X^{+}$and the spectral radius $\rho$ of $t$ is not zero. Then there exist $x_{0} \in X^{+}$and $f_{0} \in X^{*+}$ such that $x_{0} \neq 0, f_{0} \neq 0, t x_{0}=\rho x_{0}$, $t^{*} f_{0}=\rho f_{0}$.

We first prove the following variant of the Krein-Rutman theorem.

THEOREM 5. Let $t$ be a compact linear operator on a Banach space $X$, and let the spectral radius $\rho$ of $t$ be nonzero. Let $A^{+}$denote the least closed semi-algebra in $B(X)$ containing $t$ and $e$. If $A^{+} \cap\left(-A^{+}\right)=(0)$, then there exists $u \in A^{+}$such that $u \neq 0$ and $t u=\rho u$.

Proof. We use elementary techniques introduced in [2], but simplified by the fact that we are able to work throughout in $B(X)$. Suppose that $A^{+} \cap\left(-A^{+}\right)=(0)$. We note first that if $\mu>\rho$, then

$$
r_{\mu}=(\mu e-t)^{-1}=\frac{1}{\mu} e+\frac{1}{\mu^{2}} t+\frac{1}{\mu^{3}} t^{2}+\ldots \in A^{+} .
$$

We prove that there exists a sequence $\left\{\mu_{n}\right\}$ such that

$$
\mu_{n}>\rho \quad(n=1,2, \ldots), \lim _{n \rightarrow \infty} \mu_{n}=\rho, \lim _{n \rightarrow \infty}\left\|r_{\mu_{n}}\right\|=\infty .
$$

Suppose that there is no such sequence $\left\{\mu_{n}\right\}$. Then there exist positive constants $\varepsilon, M$ such that $\left\|r_{\mu}\right\| \leqq M(\rho<\mu<\rho+\varepsilon)$. We choose $\lambda, \mu$ such that

Then we have

$$
0<\lambda<\rho<\mu<\rho+\varepsilon, \quad \mu-\lambda<M^{-1} \text {. }
$$

and

$$
\begin{aligned}
r_{\lambda}=(\lambda e-t)^{-1} & =r_{\mu}+(\mu-\lambda) r_{\mu}^{2}+\ldots \in A^{+}, \\
\left(e-\frac{1}{\lambda^{n+1}} t^{n+1}\right) r_{\lambda} & =\frac{1}{\lambda} e+\frac{1}{\lambda^{2}} t+\ldots+\frac{1}{\lambda^{n+1}} t^{n} .
\end{aligned}
$$

It follows from this that

$$
r_{\lambda}=\frac{1}{\lambda^{n+1}} t^{n}+q_{n}
$$

with $q_{n} \in A^{+}$.

Let $\alpha_{n}=\lambda^{-n-1}\left\|t^{n}\right\|$. Since $0<\lambda<\rho$, we have $\lim _{n \rightarrow \infty} \alpha_{n}=\infty$, and so there exists a subsequence $\left\{\alpha_{n_{k}}\right\}$ such that

$$
\alpha_{n_{k}} \geqq \alpha_{n_{k}-1} \quad(k=1,2, \ldots) .
$$

Let $a_{k}=\lambda^{-n_{k}} t^{n_{k}-1}$. Since $\alpha_{n_{k}} \leqq \lambda^{-1}\|t\| .\left\|a_{k}\right\|$, we have $\lim _{k \rightarrow \infty}\left\|a_{k}\right\|=\infty$. By (5), we have

$$
\left\|a_{k}\right\|^{-1} r_{\lambda}=\frac{1}{\lambda} t\left(\left\|a_{k}\right\|^{-1} a_{k}\right)+\left\|a_{k}\right\|^{-1} q_{n_{k}} .
$$

By Theorem 1, there exists a subsequence of $\left\{\lambda^{-1} t\left(\left\|a_{k}\right\|^{-1} a_{k}\right)\right\}$ that converges to $b \in A^{+}$. Since the left-hand side of (7) tends to zero, the corresponding subsequence of $\left\{\left\|a_{k}\right\|^{-1} q_{n_{k}}\right\}$ 
also converges to $c \in A^{+}$, and $b+c=0$. Since $A^{+} \cap\left(-A^{+}\right)=(0)$, we conclude that $b=0$. But this is a contradiction, for (6) gives

$$
\left\|\frac{1}{\lambda} t a_{k}\right\| \geqq\left\|a_{k}\right\| \quad(k=1,2, \ldots),
$$

and so $\|b\| \geqq 1$. This contradiction proves the existence of a sequence $\left\{\mu_{n}\right\}$ satisfying (4).

Let $u_{n}=\left\|r_{\mu_{n}}\right\|^{-1} r_{\mu_{n}}$. Then $u_{n} \in A^{+},\left\|u_{n}\right\|=1$, and

$$
\left(\mu_{n} e-t\right) u_{n}=\left\|r_{\mu_{n}}\right\|^{-1} e \text {. }
$$

By Theorem 1, there exists a subsequence $\left\{u_{n_{k}}\right\}$ such that $\lim _{k \rightarrow \infty} t u_{n_{k}}=u \in A^{+}$. Then, by (4) and (8), $\lim _{k \rightarrow \infty} \mu_{n_{k}} u_{n_{k}}=u$. Therefore $\lim _{k \rightarrow \infty} \mu_{n_{k}} t u_{n_{k}}=t u$, and, since $\lim _{k \rightarrow \infty} \mu_{n_{k}}=\rho$, we have $t u=\rho u$.

Proof of the Krein-Rutman theorem. Let $X^{+}, t$ satisfy the conditions in the theorem. Each element $a$ of $A^{+}$is positive in the sense that $a X^{+} \subset X^{+}$. If $a \in A^{+} \cap\left(-A^{+}\right)$, then

$$
a X^{+} \subset X^{+} \cap\left(-X^{+}\right)=(0), a X^{+}=(0) \text {. }
$$

Since the linear hull of $X^{+}$is dense in $X$, this gives $a=0, A^{+} \cap\left(-A^{+}\right)=(0)$. Thus Theorem 5 is applicable, and there exists $u \in A^{+}$such that $u \neq 0$ and $t u=\rho u$. Again, since the linear hull of $X^{+}$is dense in $X$, there exists $x_{1} \in X^{+}$such that $u x_{1} \neq 0$. Let $x_{0}=u x_{1}$. Then $x_{0} \in X^{+}$, $x_{0} \neq 0$, and

$$
t x_{0}=t u x_{1}=\rho u x_{1}=\rho x_{0} .
$$

Also, $x_{0} \notin-X^{+}$, and $-X^{+}$is a closed positive homogeneous convex set. Therefore there exists $f_{1} \in X^{*}$ such that $f_{1}\left(x_{0}\right)=1$ and $f_{1}(x) \leqq 0\left(x \in-X^{+}\right)$. This implies that $f_{1} \in X^{*+}$. Let $f_{0}=u^{*} f_{1}$. Then $f_{0} \in X^{*+}$, and $f_{0} \neq 0$, since

$$
f_{0}\left(x_{1}\right)=\left(u^{*} f_{1}\right)\left(x_{1}\right)=f_{1}\left(u x_{1}\right)=f_{1}\left(x_{0}\right)=1 .
$$

Finally, $t^{*} u^{*}=\rho u^{*}$ (since $u t=\rho u$ ), and so

$$
t^{*} f_{0}=t^{*} u^{*} f_{1}=\rho u^{*} f_{1}=\rho f_{0} .
$$

Remark. Let $t$ satisfy the conditions of Theorem 5. If $A^{+} \cap\left(-A^{+}\right)=(0)$, then $A^{+}$is a locally compact semi-algebra. For, by Theorem 5 , the spectral radius of $t$ belongs to the spectrum of $t$, and so $A^{+}$is locally compact [3, Theorem 5]. Conversely, if $A$ is locally compact, then $A^{+} \cap\left(-A^{+}\right)$has finite dimension, since it is a normed linear space with a compact unit ball.

5. A general theorem. Given Banach spaces $X, Y$, we denote by $B(X, Y)$ the Banach space of all bounded linear mappings of $X$ into $Y$ with the usual norm.

TheORem 6. Let $X_{1}, X_{2}, X_{3}, X_{4}, Y$ be Banach spaces, let $a$ and $d$ be compact linear mappings of $X_{1}$ into $X_{2}$ and $X_{3}$ into $X_{4}$ respectively, let $b$ and $c$ be bounded linear mappings of $Y$ into $B\left(X_{2}, X_{4}\right)$ and $B\left(X_{1}, X_{3}\right)$ respectively, and let

$$
Z=\{y \in Y:(b y) \circ a=d \circ(c y)\} .
$$


Then the mapping $z \rightarrow(b z) \circ a$ is a compact linear mapping of $Z$ into $B\left(X_{1}, X_{4}\right)$.

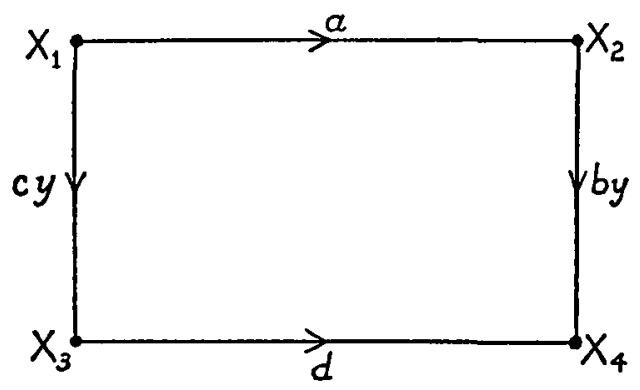

Proof. Let $U, V, W$ be the closed balls given by

$$
U=\left\{x \in X_{1}:\|x\| \leqq 1\right\}, \quad V=\{z \in Z:\|z\| \leqq 1\}, \quad W=\left\{x \in X_{3}:\|x\| \leqq\|c\|\right\},
$$

and let $E=\overline{a U}, F=\overline{d W}$. Then $E$ and $F$ are compact subsets of $X_{2}$ and $X_{4}$ in their norm topologies, and we have

$$
(c z) x \in W \quad(x \in U, z \in V) \text {, }
$$

from which

$$
((b z) \circ a) x=(d \circ(c z)) x \in d W \quad(x \in U, z \in V) .
$$

Since $b z$ is continuous, it follows that

$$
(b z) x \in F \quad(x \in E, z \in V) \text {. }
$$

Thus, for each $x \in E$, the image of $V$ under the mapping $z \rightarrow(b z) x$ is contained in a compact subset of $X_{4}$. Also, $\{b z: z \in V\}$ is an equicontinuous family of mappings of the compact space $E$ into the Banach space $X_{4}$. Therefore, by Ascoli's theorem for Banach space valued functions, given $z_{n} \in V(n=1,2, \ldots)$, there exists a subsequence $\left\{z_{n_{k}}\right\}$ such that $\left\{\left(b z_{n_{k}}\right) x\right\}$ converges uniformly on $E$. Since $a U \subset E$, it follows that $\left(\left(b z_{n_{k}}\right) \circ a\right) x$ converges uniformly on $U$, i.e. $\left\{\left(b z_{n_{k}}\right) \circ a\right\}$ converges with respect to the norm in $B\left(X_{1}, X_{4}\right)$.

Example 1. Let $t$ be a compact linear operator on a Banach space $X$ over $\mathbf{F}$, where $\mathbf{F}$ is either $\mathbf{R}$ or $\mathbf{C}$. Take $X_{1}=X_{2}=X, X_{3}=X_{4}=\mathrm{F}, Y=X^{*}$, the dual space of $X$. Let $a=t$, $c=t^{*}$, and let $b$ and $d$ be the identity operators on $X^{*}$ and $\mathbf{F}$ respectively. Since

$$
(b y) \circ a=y \circ t=t^{*} y=d \circ(c y) \quad(y \in Y),
$$

we have $Z=Y=X^{*}$. Thus the mapping $y \rightarrow t^{*} y$ is a compact linear mapping of $X^{*}$ into $B\left(X_{1}, X_{4}\right)=B(X, \mathbf{F})=X^{*}$. This is Schauder's theorem.

Example 2. Let $t$ be a compact linear operator on a Banach space $X$, let $X_{1}=X_{2}=$ $X_{3}=X_{4}=X$, and let $Y=B(X, X)$. Let $a=d=t$, and let both $b$ and $c$ be the identity operator 
on $Y$. Then

$$
Z=\{s \in B(X, X): s t=t s\}
$$

and $s \rightarrow s t$ is a compact linear mapping of $Z$ into itself. This is Theorem 1 .

Example 3. Let $X_{1}, X_{2}, X_{3}, X_{4}$ be Banach spaces, and let $Y=B\left(X_{2}, X_{3}\right)$. Let $a$ and $d$ be compact linear mappings of $X_{1}$ into $X_{2}$ and $X_{3}$ into $X_{4}$ respectively and let $b$ and $c$ be defined by $b y=d \circ y, c y=y \circ a$. Then

$$
(b y) \circ a=d \circ y \circ a=d \circ(c y) \quad(y \in Y) .
$$

Therefore $Z=Y=B\left(X_{2}, X_{3}\right)$, and the mapping $y \rightarrow d \circ y \circ a$ is a compact linear mapping of $B\left(X_{2}, X_{3}\right)$ into $B\left(X_{1}, X_{4}\right)$. This result has been proved by $\mathrm{K}$. Vala [9] as an application of his formulation of Ascoli's theorem.

\section{REFERENCES}

I. J. C. Alexander, Banach algebras of compact operators; to appear.

2. F. F. Bonsall, Linear operators in complete positive cones, Proc. London Math. Soc. (3) 8 (1958), 53-75.

3. F. F. Bonsall and B. J. Tomiuk, The semi-algebra generated by a compact linear operator, Proc. Edinburgh Math. Soc. (2) 14 (1965), 177-195.

4. F. F. Bonsall and J. Duncan, Dual representations of Banach algebras, Acta Math.; to appear.

5. M. G. Krein and M. A. Rutman, Linear operators leaving invariant a cone in a Banach space (Russian), Uspehi Mat. Nauk (N.S.) 3, No. 1 (23) (1948), 3-95. English translation: American Math. Soc. Translation 26.

6. F. Riesz, Uber lineare Functionalgleichungen, Acta Math. 41 (1918), 71-98.

7. C. E. Rickart, General theory of Banach algebras (Van Nostrand, 1960).

8. J. Schauder, Uber lineare, vollstetige Functionaloperationen, Studia Math. 2 (1930), 183-196.

9. K. Vala, On compact sets of compact operators, Ann. Acad. Sci. Fenn. Ser. A.I. No 351 (1964), $9 \mathrm{pp}$.

\section{UNIVERSITY OF EDINBURGH}

